\title{
RELATIVE LOCATIONS OF SUBWORDS IN FREE OPERATED SEMIGROUPS AND MOTZKIN WORDS
}

\author{
LI GUO AND SHANGHUA ZHENG
}

\begin{abstract}
AвSTRACT. Bracketed words are basic structures both in mathematics (such as Rota-Baxter algebras) and mathematical physics (such as rooted trees) where the locations of the substructures are important. In this paper we give the classification of the relative locations of two bracketed subwords of a bracketed word in an operated semigroup into the separated, nested and intersecting cases. We achieve this by establishing a correspondence between relative locations of bracketed words and those of words by applying the concept of Motzkin words which are the algebraic forms of Motzkin paths.
\end{abstract}

\section{Contents}

1. Introduction

2. Relative locations of subwords

2.1. Subwords

2.2. Substrings

3. Bracketed words and Motzkin words

3.1. Bracketed words

3.2. Motzkin words

3.3. $\vec{\star}$-bracketed words and $\vec{\star}$-Motzkin words

4. Relative locations in bracketed words and Motzkin words

4.1. Placements in bracketed words and Motzkin words

4.2. Relationship between relative locations

4.3. Relative locations of bracketed subwords

References

\section{INTRODUCTION}

As a basic property of sets, there are three relative locations of any two subsets of a given set: separated (disjoint), nested (including) and intersecting (overlapping). See the proof of Theorem 2.11 for example. Similarly, there are three relative locations of two subwords in a given word, a property that is essential in rewriting systems (critical pairs) and Gröbner bases [2]. Analogous classification of relative locations of combinatorial objects, such as Feynman graphs,

Date: June 28, 2018.

2010 Mathematics Subject Classification. 20M05, 20M99, 05E15, 16S15, 08B20.

Key words and phrases. bracketed word, relative locations, operated semigroup, Motzkin word, Motskin path, rooted tree

Corresponding author: Li Guo, Department of Mathematics and Computer Science, Rutgers University, Newark, NJ

07102, USA; E-mail: liguo@ rutgers.edu; Phone: 973-353-3917; Fax: 973-353-5270. 
plays an important role in combinatorics and physics, for example in the renormalization of quantum field theory [3, 9, 14, 15]].

The classification of relative locations can be quite subtle in some structures, especially when a non-identity operator is present, such as in differential algebras, Rota-Baxter algebras [12] and, more generally, operated algebras [5, 7, 13, 17]. Further by [11] free operated semigroups have natural combinatorial presentation as rooted trees which serve as the baby models for Feynman graphs [8, 14]. Considering the importance of such classification in the study of these mathematics and physics structures, especially their Gröbner-Shirshov bases (compositions and diamond lemma) [月, 6], it is crucial to establish such a classification. In this paper, we give an explicit correspondence between relative locations of two bracketed subwords and those of Motzkin words and subwords. As a direct consequence, we obtain the classification of the relative locations of bracketed subwords.

We take two steps in our treatment to deal with two subtle points of bracketed words. In Section $€$ we deal with the first subtle point which is already present in studying relative locations of two subwords in comparison with two subsets, namely that one subword can appear at multiple locations in a given word. The concept of a $\star$-word [7] allows us to give a unique label to each appearance of a subword, called a placement. We then show that each placement corresponds uniquely to a substring of the string corresponding to the given word, converting the problem of studying subwords to that of subsets which can be solved easily as mentioned above. The second subtle point arise when we deal with bracketed words in Section 3 since the action of the bracket together with the product of the word gives the bracketed words a quite complicated structure. To resolve this difficulty, we make use of a bijection introduced in [11] between bracketed words and a class of words called Motzkin words on a larger set. We then show in Section 7 that this bijection preserves the relative locations of the (bracketed) word, reducing the study of relative locations of bracketed words to the one of words for which we can apply Section 2 .

\section{RELATIVE LOCATIONS OF SUBWORDS}

In this section, we consider the relative locations of two subwords of a fixed word. This serves as both the prototype and preparation for our study of the relative locations of two bracketed subwords in later sections.

\subsection{Subwords.}

Definition 2.1. Let $Z$ be a set. Let $M(Z)$ be the free monoid on $Z$ consisting of words in the alphabet set $Z$. Thus a word is either the empty word 1 or of the form $w=z_{1} \cdots z_{n}, z_{i} \in Z$, $1 \leqslant i \leqslant n$. A subword of $w$ is defined to be a word $u \neq 1$ that is a part of $w$. Let $S(Z):=M(Z) \backslash\{1\}$ be the free semigroup on $Z$.

We emphasize that the empty word 1 is not taken to be a subword in this paper.

Note that a subword $u$ of $w \in M(Z)$ may appear in $w$ at multiple locations. For example, for $z \in Z, u:=z$ appears in $w:=z z z$ at three different locations. It is often important to be precise about the location of a subword. This is the case, for example, in the study of rewriting systems and Gröbner-Shirshov bases [2, 7, 13, 17]. For this purpose, we need additional information for the location of the subword $u$. Such information is provided by the concept of $\star$-words [7].

Definition 2.2. Let $Z$ be a set.

(a) Let $\star$ be an element not in $Z$. A $\star$-word on $Z$ is a word in $M(Z \cup\{\star\})$ in which $\star$ appears exactly once. The set of $\star$-words on $Z$ is denoted by $M^{\star}(Z)$. 
(b) More generally, for $\star_{1}, \cdots, \star_{k}$ not in $Z$, denote $\vec{\star}=\left(\star_{1}, \cdots, \star_{k}\right)$. A $\vec{\star}$-word on $Z$ is a word in $M\left(Z \cup\left\{\star_{1}, \cdots, \star_{k}\right\}\right)$ in which $\star_{i}$ appears exactly once for each $i=1, \cdots, k$. The set of $\vec{\star}$-words is denoted by $M^{\vec{\star}}(Z)$. When $k=2$, it is denoted by $M^{\star_{1}, \star_{2}}(Z)$.

(c) For $p \in M^{\vec{\star}}(Z)$ and $u_{1}, \cdots, u_{k} \in M(Z)$, let $\left.p\right|_{u_{1}, \cdots, u_{k}}=\left.p\right|_{\star_{1} \mapsto u_{1}, \cdots, \star_{k} \mapsto u_{k}}$ denote the word in $M(Z)$ when the $\star_{i}$ in $p$ is replaced by $u_{i}$, where $i=1, \cdots, k$.

Now we can be more precise on a particular appearance of a subword.

Definition 2.3. Let $u, w \in M(Z)$ be words on $Z$. A placement of $u$ in $w$ (by $p)$ is a pair $(u, p)$ where $p$ is in $M^{\star}(Z)$ such that $\left.p\right|_{u}=w$.

Thus $\left(u_{1}, p_{1}\right)=\left(u_{2}, p_{2}\right)$ means $u_{1}=u_{2}$ and $p_{1}=p_{2}$.

Of course $u$ is a subword of $w$ if there is $p \in M^{\star}(Z)$ such that $(u, p)$ is a placement of $u$ in $w$. However, the usefulness of the placement notion is its role in distinguishing different appearances of $u$ in $w$. For example, the three appearances of $u=z$ in $w=z z z$ are identified by the three placements $\left(u, p_{1}\right),\left(u, p_{2}\right)$ and $\left(u, p_{3}\right)$ where $p_{1}=\star z z, p_{2}=z \star z$ and $p_{3}=z z \star$.

The concept of a placement is also essential in determining the relative locations of two subwords of a given word.

Example 2.4. Let $Z=\{x, y\}$ and let $w=x y x y x y$. Then $u:=x y x$ appears at two locations in $w$ and $v:=x y$ appears at three locations, as shown in the following equation.

$$
w=\underbrace{x y x}_{(u, p)} y x y=\overbrace{x y}^{\left(v, q_{1}\right)} \overbrace{x y}^{\left(v, q_{2}\right)} \overbrace{x y}^{\left(v, q_{3}\right)} .
$$

Take $(u, p)$ to be the first appearance (from the left) of $u$ in $w$. Thus $p=\star y x y$. Then the three placements of $v=x y$ in $w$, given by $\left(v, q_{i}\right), i=1,2,3$, with $q_{1}=\star x y x y, q_{2}=x y \star x y$ and $\left.q_{3}=x y x y \star\right)$. These three placements of $v$ are in three different kinds of relative locations with respect to $u$ : the left $v$ (in $\left.\left(v, q_{1}\right)\right)$ is a subword of $u$, the middle $v$ (in $\left(v, q_{2}\right)$ ) is not a subword of $u$ but has a nonempty intersection with $u$, and the right $v$ (in $\left.\left(v, q_{3}\right)\right)$ is disjoint with $u$.

This situation can again be made precise by $\star$-words.

Definition 2.5. Let $w$ be a word in $M(Z)$. Two placements $\left(u_{1}, p_{1}\right)$ and $\left(u_{2}, p_{2}\right)$ are called

(a) separated if there exists an element $p$ in $M^{\star_{1}, \star_{2}}(Z)$ such that $w=\left.p\right|_{u_{1}, u_{2}},\left.p_{1}\right|_{\star_{1}}=\left.p\right|_{\star_{1}, u_{2}}$ and $\left.p_{2}\right|_{\star_{2}}=\left.p\right|_{u_{1}, \star_{2}}$

(b) nested if there exists an element $p$ in $M^{\star}(Z)$ such that $p_{1}=\left.p_{2}\right|_{p}$, or $p_{2}=\left.p_{1}\right|_{p}$;

(c) intersecting if there exist an element $p$ in $M^{\star}(Z)$ and elements $a, b, c$ in $S(Z)$ such that

(i) either $w=\left.p\right|_{a b c}, p_{1}=\left.p\right|_{\star c}, p_{2}=\left.p\right|_{a \star}$;

(ii) or $w=\left.p\right|_{a b c}, p_{1}=\left.p\right|_{a \star}, p_{2}=\left.p\right|_{\star c}$.

Example 2.6. With the $w, u$ and the three appearances of $v$ in Example 2.4, we have the corresponding placements $(u, p)$ with $p=\star y x y$, and $\left(v, q_{1}\right),\left(v, q_{2}\right)$ and $\left(v, q_{3}\right)$. Then $(u, p)$ and $\left(v, q_{1}\right)$ (resp. $\left(v, q_{2}\right)$, resp. $\left.\left(v, q_{3}\right)\right)$ are nested (resp. intersecting, resp. separated).

2.2. Substrings. We now give another description of a placement of a subword that makes it easier to classify the relative locations of two subwords. Denote $[n]:=\{1, \cdots, n\}$ and $[i, k]:=$ $\{i, \cdots, k\}$ for $n, i \geqslant 1, k \geqslant i$.

Let $Z$ be a set. Let $w=z_{1} \cdots z_{n}, z_{i} \in Z, 1 \leqslant i \leqslant n$, be a word in $M(Z)$. Let $p:=(u, p)$ be a placement of $u$ in $w$. Then $p$ is of the form $p=x_{1} \cdots x_{j} \star x_{j+1} \cdots x_{m}$ with $x_{i} \in Z, 1 \leqslant i \leqslant m$, and 
$u=y_{1} \cdots y_{t}$ with $y_{j} \in Z, 1 \leqslant j \leqslant t$. Comparing

$$
x_{1} \cdots x_{j} y_{1} \cdots y_{t} x_{j+1} \cdots x_{m}=\left.p\right|_{u}=w=z_{1} \cdots z_{n}
$$

in the free monoid $M(Z)$, we obtain $p=z_{1} \cdots z_{j-1} \star z_{k+1} \cdots z_{n}$ for unique $j=j_{\mathfrak{p}}$ and $k=k_{\mathfrak{p}}$. Thus $u=z_{j} \cdots z_{k}$.

Definition 2.7. With notations as above, the set $I_{\mathfrak{p}}:=\left[j_{\mathfrak{p}}, k_{\mathfrak{p}}\right]$ is called the location of the placement $\mathfrak{p}=(u, p)$ in $w$. Denote

$$
\mathcal{L} \mathcal{O}(w):=\{[j, k] \mid 1 \leqslant j \leqslant k \leqslant n\} .
$$

Also denote

$$
\mathcal{P} \mathcal{L}(w):=\{(u, p) \mid(u, p) \text { is a placement in } w\} .
$$

Proposition 2.8. Let $1 \neq w \in M(Z)$. The map

$$
\eta: \mathcal{P} \mathcal{L}(w) \longrightarrow \mathcal{L} \mathcal{O}(w), \quad \mathfrak{p}=(u, p) \mapsto\left[j_{\mathfrak{p}}, k_{\mathfrak{p}}\right],
$$

is a bijection.

Proof. Let $w=z_{1} \cdots z_{n}$. Given a placement $(u, p)$ in $w$. Then $p=z_{1} \cdots z_{j-1} \star z_{k+1} \cdots z_{n} \in M^{\star}(Z)$ and $u=z_{j} \cdots z_{k}, 1 \leqslant j \leqslant k \leqslant n$. Thus if $\mathfrak{p}_{1}=\left(u_{1}, p_{1}\right)$ and $\mathfrak{p}_{2}=\left(u_{2}, p_{2}\right)$ are in $\mathcal{P} \mathcal{L}(w)$ and $\mathfrak{p}_{1} \neq \mathfrak{p}_{2}$, then we have $p_{1} \neq p_{2}$. Hence either $j_{\mathfrak{p}_{1}} \neq j_{\mathfrak{p}_{2}}$ or $k_{\mathfrak{p}_{1}} \neq k_{\mathfrak{p}_{2}}$. Then $\eta\left(u_{1}, p_{1}\right) \neq \eta\left(u_{2}, p_{2}\right)$. Hence $\eta$ is injective.

Further for any $[j, k] \in \mathcal{L} \mathcal{O}(w)$, define $u=z_{j} \cdots z_{k}$ and $p=z_{1} \cdots z_{j-1} \star z_{k+1} \cdots z_{n}$. Then we have $\left.p\right|_{u}=w$ and $\eta(u, p)=[j, k]$. Hence $\eta$ is surjective.

Definition 2.9. Two nonempty subsets $I$ and $J$ of $[n]$ are called

(a) separated if $I \cap J=\emptyset$;

(b) nested if $I \subseteq J$ or $J \subseteq I$;

(c) intersecting if $I \cap J \neq \emptyset, I \nsubseteq J$ and $J \nsubseteq I$.

Consider the $w, u$ and $v$ in Example 2.4 and their corresponding placements $(u, p),\left(v, q_{1}\right),\left(v, q_{2}\right)$ and $\left(v, q_{3}\right)$ in Example 2.6. The locations of the placements are $\eta(u, p)=[1,3]$ and $\eta\left(v, q_{1}\right)=$ $[1,2], \eta\left(v, q_{2}\right)=[3,4]$ and $\eta\left(v, q_{3}\right)=[5,6]$. Then we see that, as subsets of [6], $[1,3]$ and $[1,2]$ (resp. [3, 4], resp. [5,6]) are also nested (resp. intersecting, resp. separated). The next theorem shows that this equivalence holds in general.

Theorem 2.10. Let $Z$ be a set. Let $1 \neq w=z_{1} \cdots z_{n}$ be in $M(Z)$ where $z_{i} \in Z, 1 \leqslant i \leqslant n$. Then placements $\mathfrak{p}_{1}=\left(u_{1}, p_{1}\right)$ and $\mathfrak{p}_{2}=\left(u_{2}, p_{2}\right)$ in $w$ are separated (resp. nested, resp. intersecting) if and only if the corresponding subsets $I_{\mathfrak{p}_{1}}$ and $I_{\mathfrak{p}_{2}}$ of $[n]$ are separated (resp. nested, resp. intersecting).

Proof. First suppose that the placements $\left(u_{1}, p_{1}\right)$ and $\left(u_{2}, p_{2}\right)$ in $w$ are separated. Then there exists an element $p$ in $M^{\star_{1}, \star_{2}}(Z)$ such that

$$
w=\left.p\right|_{u_{1}, u_{2}},\left.\quad p_{1}\right|_{\star_{1}}=\left.p\right|_{\star_{1}, u_{2}},\left.\quad p_{2}\right|_{\star_{2}}=\left.p\right|_{u_{1}, \star_{2}} .
$$

From $\left.p\right|_{u_{1}, u_{2}}=z_{1} \cdots z_{n}$, there are $1 \leqslant j_{1} \leqslant k_{1}<j_{2} \leqslant k_{2} \leqslant n$ such that

$$
p=z_{1} \cdots z_{j_{1}-1} \star_{1} z_{k_{1}+1} \cdots z_{j_{2}-1} \star_{2} z_{k_{2}+1} \cdots z_{n},
$$

or

$$
p=z_{1} \cdots z_{j_{1}-1} \star_{2} z_{k_{1}+1} \cdots z_{j_{2}-1} \star_{1} z_{k_{2}+1} \cdots z_{n} .
$$


Thus we have $\left\{I_{\mathfrak{p}_{1}}, I_{\mathfrak{p}_{2}}\right\}=\left\{\left[j_{1}, k_{1}\right],\left[j_{2}, k_{2}\right]\right\}$ and hence $I_{\mathfrak{p}_{1}} \cap I_{\mathfrak{p}_{2}}=\emptyset$. Conversely, suppose $I_{\mathfrak{p}_{1}} \cap I_{\mathfrak{p}_{2}}=$ $\emptyset$. Let $I_{\mathfrak{p}_{1}}=\left[j_{1}, k_{1}\right]$ and $I_{\mathfrak{p}_{2}}=\left[j_{2}, k_{2}\right]$. Then we have $k_{1}<j_{2}$ or $k_{2}<j_{1}$. Without loss of generality, assume $k_{1}<j_{2}$. Then take $u_{1}=z_{j_{1}} \cdots z_{k_{1}}$ and $u_{2}=z_{j_{2}} \cdots z_{k_{2}}$. Define $p=z_{1} \cdots z_{j_{1}-1} \star_{1}$ $z_{k_{1}+1} \cdots z_{j_{2}-1} \star_{2} z_{k_{2}+1} \cdots z_{n}$. Then we have

$$
w=\left.p\right|_{u_{1}, u_{2}},\left.\quad p_{1}\right|_{\star_{1}}=\left.p\right|_{\star_{1}, u_{2}},\left.\quad p_{2}\right|_{\star_{2}}=\left.p\right|_{u_{1}, \star_{2}},
$$

as needed.

Next suppose that $\left(u_{1}, p_{1}\right)$ and $\left(u_{2}, p_{2}\right)$ are nested. Then there exists an element $p$ in $M^{\star}(Z)$ such that $p_{1}=\left.p_{2}\right|_{p}$ or $p_{2}=\left.p_{1}\right|_{p}$. Without loss of generality, we can assume $p_{1}=\left.p_{2}\right|_{p}$. From $w=\left.p_{1}\right|_{u_{1}}$ and $w=\left.p_{2}\right|_{u_{2}}$ we obtain

$$
z_{1} \cdots z_{j_{1}-1} \star z_{k_{1}+1} \cdots z_{n}=p_{1}=\left.p_{2}\right|_{p}=\left.z_{1} \cdots z_{j_{2}-1} \star\right|_{p} z_{k_{2}+1} \cdots z_{n} .
$$

Thus $j_{2} \leqslant j_{1} \leqslant k_{1} \leqslant k_{2}$ and hence $I_{\mathfrak{p}_{1}} \subseteq I_{\mathfrak{p}_{2}}$. Conversely, suppose $I_{\mathfrak{p}_{1}}$ and $I_{\mathfrak{p}_{2}}$ are nested. We may assume that $I_{\mathfrak{p}_{1}}=\left[j_{1}, k_{1}\right] \subseteq I_{\mathfrak{p}_{2}}=\left[j_{2}, k_{2}\right]$. Then define $p=z_{j_{2}} \cdots z_{j_{1}-1} \star z_{k_{1}+1} \cdots z_{k_{2}}$, with the convention that $p=\star z_{k_{1}+1} \cdots z_{k_{2}}$ if $j_{1}=j_{2}$ and $p=z_{j_{2}} \cdots z_{j_{1}-1} \star$ if $k_{1}=k_{2}$. We have $\left.p_{2}\right|_{p}=p_{1}$. Hence $\left(u_{1}, p_{1}\right)$ and $\left(u_{2}, p_{2}\right)$ are nested.

Finally, suppose that $\left(u_{1}, p_{1}\right)$ and $\left(u_{2}, p_{2}\right)$ are intersecting. Then there exist $p$ in $M^{\star}(Z)$ and $a, b, c$ in $S(Z)$ such that

$$
w=\left.p\right|_{a b c}, \quad p_{1}=\left.p\right|_{\star c}, \quad p_{2}=\left.p\right|_{a \star},
$$

or

$$
w=\left.p\right|_{a b c}, \quad p_{1}=\left.p\right|_{a \star}, \quad p_{2}=\left.p\right|_{\star c} .
$$

We just need to consider the first case since the proof of the second case is similar. Denote $p=z_{1} \cdots z_{j-1} \star z_{k+1} \cdots z_{n}$. From

$$
z_{1} \cdots z_{j_{1}-1} \star z_{k_{1}+1} \cdots z_{n}=p_{1}=\left.p\right|_{\star c}=z_{1} \cdots z_{j-1} \star c z_{k+1} \cdots z_{n}
$$

we obtain $j_{1}=j$ and $c=z_{k_{1}+1} \cdots z_{k}$. Since $c \neq 1$, we have $k_{1}<k$. Similarly, from

$$
z_{1} \cdots z_{j_{2}-1} \star z_{k_{2}+1} \cdots z_{n}=p_{2}=\left.p\right|_{a \star}=z_{1} \cdots z_{j-1} a \star z_{k+1} \cdots z_{n}
$$

we obtain $k_{2}=k$ and $a=z_{j} \cdots z_{j_{2}-1}$. Since $a \neq 1$, we have $j<j_{2}$. Consequently,

$$
w=\left.p\right|_{a b c}=z_{1} \cdots z_{j-1} a b c z_{k+1} \cdots z_{n}=z_{1} \cdots z_{j_{2}-1} b z_{k_{1}+1} \cdots z_{n} .
$$

Since $b \neq 1$, we have $j_{2} \leqslant k_{1}$. Then

$$
j_{1}=j<j_{2} \leqslant k_{1}<k=k_{2}
$$

Thus

$$
I_{\mathfrak{p}_{1}} \cap I_{\mathfrak{p}_{2}}=\left[j_{2}, k_{1}\right] \neq \emptyset, \quad I_{\mathfrak{p}_{1}} \backslash I_{\mathfrak{p}_{2}}=\left[j_{1}, j_{2}-1\right] \neq \emptyset, \quad I_{\mathfrak{p}_{2}} \backslash I_{\mathfrak{p}_{1}}=\left[k_{1}+1, k_{2}\right] \neq \emptyset .
$$

Hence $I_{\mathfrak{p}_{1}} \nsubseteq I_{\mathfrak{p}_{2}}$ and $I_{\mathfrak{p}_{2}} \nsubseteq I_{\mathfrak{p}_{1}}$. Thus $I_{\mathfrak{p}_{1}}$ and $I_{\mathfrak{p}_{2}}$ are intersecting.

Conversely, suppose that $I_{\mathfrak{p}_{1}} \cap I_{\mathfrak{p}_{2}} \neq \emptyset, I_{\mathfrak{p}_{1}} \nsubseteq I_{\mathfrak{p}_{2}}$ and $I_{\mathfrak{p}_{2}} \nsubseteq I_{\mathfrak{p}_{1}}$. From $I_{\mathfrak{p}_{1}} \cap I_{\mathfrak{p}_{2}} \neq \emptyset$, we have $j_{1} \leqslant k_{2}$ and $j_{2} \leqslant k_{1}$. Then from $I_{\mathfrak{p}_{1}} \nsubseteq I_{\mathfrak{p}_{2}}$ and $I_{\mathfrak{p}_{2}} \nsubseteq I_{\mathfrak{p}_{1}}$, we have $k_{1}>k_{2}$ and $j_{1}>j_{2}$, or $k_{1}<k_{2}$ and $j_{1}<j_{2}$. We just consider the first case with the second case being similar. Then we have $j_{2}<j_{1} \leqslant k_{2}<k_{1}$. Define

$$
p=z_{1} \cdots z_{j_{2}-1} \star z_{k_{1}+1} \cdots z_{n}, \quad a=z_{j_{2}} \cdots z_{j_{1}-1}, \quad b=z_{j_{1}} \cdots z_{k_{2}}, \quad c=z_{k_{2}+1} \cdots z_{k_{1}} .
$$

Then we have

$$
a, b, c \neq 1, \quad w=\left.p\right|_{a b c}, \quad p_{2}=\left.p\right|_{\star c}, \quad p_{1}=\left.p\right|_{a \star} .
$$

This shows that $\left(u_{1}, p_{1}\right)$ and $\left(u_{2}, p_{2}\right)$ are intersecting. 
The following result is the classification of the relative locations of two placements in the free monoid $M(Z)$. Its generalization to operated monoids will be treated in the subsequent sections.

Theorem 2.11. Let $1 \neq w$ be a word in $M(Z)$. For any two placements $\mathfrak{p}_{1}=\left(u_{1}, p_{1}\right)$ and $\mathfrak{p}_{2}=$ $\left(u_{2}, p_{2}\right)$ in $w$, exactly one of the following is true:

(a) $\left(u_{1}, p_{1}\right)$ and $\left(u_{2}, p_{2}\right)$ are separated;

(b) $\left(u_{1}, p_{1}\right)$ and $\left(u_{2}, p_{2}\right)$ are nested;

(c) $\left(u_{1}, p_{1}\right)$ and $\left(u_{2}, p_{2}\right)$ are intersecting.

Proof. Let $w=z_{1} \cdots z_{n}$ with $z_{1}, \cdots, z_{n} \in Z, n \geqslant 1$. By Theorem 2.10, we only need to prove that the same conclusion holds for $I_{\mathfrak{p}_{1}}$ and $I_{\mathfrak{p}_{2}}$. But this follows from the simple fact that, for the two subsets $I_{\mathfrak{p}_{1}}$ and $I_{\mathfrak{p}_{2}}$ of $[n]$, exactly one of the following is true:

(a) $I_{\mathfrak{p}_{1}}$ and $I_{\mathfrak{p}_{2}}$ have empty intersection ( $\Leftrightarrow I_{\mathfrak{p}_{1}}$ and $I_{\mathfrak{p}_{2}}$ are separated);

(b) $I_{\mathfrak{p}_{1}}$ and $I_{\mathfrak{p}_{2}}$ have nonempty intersection, and $I_{\mathfrak{p}_{1}} \subseteq I_{\mathfrak{p}_{2}}$ or $I_{\mathfrak{p}_{2}} \subseteq I_{\mathfrak{p}_{1}}\left(\Leftrightarrow I_{\mathfrak{p}_{1}}\right.$ and $I_{\mathfrak{p}_{2}}$ are nested);

(c) or $I_{\mathfrak{p}_{1}}$ and $I_{\mathfrak{p}_{2}}$ have nonempty intersection, and $I_{\mathfrak{p}_{1}} \nsubseteq I_{\mathfrak{p}_{2}}$ and $I_{\mathfrak{p}_{2}} \nsubseteq I_{\mathfrak{p}_{1}}\left(\Leftrightarrow I_{\mathfrak{p}_{1}}\right.$ and $I_{\mathfrak{p}_{2}}$ are intersecting).

\section{BRACKeTED WORdS AND MOTZKIN WORDS}

We recall the concepts of bracketed words and Motzkin words [1, 11, 16, 13] before generalizing Theorem 2.11 to this context.

3.1. Bracketed words. We first recall the concept and construction of free operated monoids, following [11, 13].

Definition 3.1. An operated monoid is a monoid $U$ together with a map $P: U \rightarrow U$. A morphism from an operated monoid $U$ with a map $P: U \rightarrow U$ to an operated monoid $V$ with a map $Q: V \rightarrow V$ is a monoid homomorphism $f: U \rightarrow V$ such that $f \circ P=Q \circ f$.

Definition 3.2. A free operated monoid on a set $Y$ is an operated monoid $\left(U_{Y}, P_{Y}\right)$ together with a map $j_{Y}: Y \rightarrow U_{Y}$ with the property that, for any operated monoid $(V, Q)$ and any map $f: Y \rightarrow V$, there is a unique morphism $\bar{f}:\left(U_{Y}, P_{Y}\right) \rightarrow(V, Q)$ of operated monoids such that $f=\bar{f} \circ j_{Y}$.

For any set $Y$, let $\lfloor Y\rfloor:=\{\lfloor y\rfloor \mid y \in Y\}$ denote a set indexed by, but disjoint from $Y$. Let $X$ be a given set. We will construct a direct system $\left\{\mathfrak{M}_{n}:=\mathfrak{M}_{n}(X)\right\}_{n \geqslant 0}$ of monoids with natural embeddings $\widetilde{i}_{n-1}: \mathfrak{M}_{n-1} \hookrightarrow \mathfrak{M}_{n}$ for $n \geqslant 1$ by induction on $n$. The free operated monoid $\mathfrak{M}(X)$ on $X$ is the direct limit of the system, after we equip it with a natural operator.

First let $\mathfrak{M}_{0}:=M(X)$. Then define the monoid

$$
\mathfrak{M}_{1}:=M\left(X \cup\left\lfloor\mathfrak{M}_{0}\right\rfloor\right)=M(X \cup\lfloor M(X)\rfloor)
$$

and denote the natural embedding of monoids induced by the inclusion $X \hookrightarrow X \cup\left\lfloor\mathfrak{M}_{0}\right\rfloor$ by

$$
{\widetilde{i_{0}}}_{0}: \mathfrak{M}_{0}=M(X) \hookrightarrow M\left(X \cup\left\lfloor\mathfrak{M}_{0}\right\rfloor\right)=\mathfrak{M}_{1} .
$$

Inductively assuming that $\mathfrak{M}_{n}$ and $\widetilde{i}_{n-1}: \mathfrak{M}_{n-1} \hookrightarrow \mathfrak{M}_{n}$ have been defined for $n \geqslant 1$, we define the monoid

$$
\mathfrak{M}_{n+1}:=M\left(X \cup\left\lfloor\mathfrak{M}_{n}\right\rfloor\right)
$$


From the embedding of monoids $\widetilde{i}_{n-1}: \mathfrak{M}_{n-1} \hookrightarrow \mathfrak{M}_{n}$, we have an embedding $\left\lfloor\mathfrak{M}_{n-1}\right\rfloor \hookrightarrow\left\lfloor\mathfrak{M}_{n}\right\rfloor$. By the freeness of $\mathfrak{M}_{n}=M\left(X \cup\left\lfloor\mathfrak{M}_{n-1}\right\rfloor\right)$, we obtain a natural embedding of monoids

$$
\widetilde{i}_{n}: \mathfrak{M}_{n}=M\left(X \cup\left\lfloor\mathfrak{M}_{n-1}\right\rfloor\right) \hookrightarrow M\left(X \cup\left\lfloor\mathfrak{M}_{n}\right\rfloor\right)=\mathfrak{M}_{n+1} \text {. }
$$

This completes our inductive definition of the direct system. Let

$$
\mathfrak{M}(X):=\bigcup_{n \geqslant 0} \mathfrak{M}_{n}=\lim _{\longrightarrow} \mathfrak{M}_{n}
$$

be the direct limit of the system. We note that $\mathfrak{M}(X)$ is a monoid. By taking direct limit on both sides of $\mathfrak{M}_{n}=M\left(X \cup\left\lfloor\mathfrak{M}_{n-1}\right\rfloor\right)$, we obtain

$$
\mathfrak{M}(X)=M(X \cup\lfloor\mathfrak{M}(X)\rfloor)
$$

whose elements are called bracketed words.

The depth of $f \in \mathfrak{M}(X)$ is defined to be

$$
\operatorname{depth}(f):=\min \left\{n \mid f \in \mathfrak{M}_{n}\right\} .
$$

The following result shows that $\mathfrak{M}(X)$ is the equivalence of free monoids in the category of operated monoids.

Lemma 3.3. [11] Define the map \lfloor\rfloor$: \mathfrak{M}(X) \rightarrow \mathfrak{M}(X)$ by taking $w \in \mathfrak{M}(X)$ to $\lfloor w\rfloor$. Let $j_{X}: X \rightarrow$ $\mathfrak{M}(X)$ be the natural embedding. Then the triple $\left(\mathfrak{M}(X),\llcorner\rfloor, j_{X}\right)$ is the free operated monoid on $X$.

By [11, Theorem 4.2], another representation of free operated semigroups is given by rooted trees. See [0, 14] for the application of rooted trees in quantum field theory.

3.2. Motzkin words. We now recall the definition of Motzkin words which aquired its name since it encodes Motzkin paths [10]. Motzkin words give another construction of free operated monoids [11] and in this paper serve as the bridge between bracketed words and the usual words.

Let $X$ be a set. Let $\Perp$ and $\Perp$ be symbols not in $X$.

Definition 3.4. An element of the free monoid $M(X \cup\{\Perp, \Downarrow\})$ is called a Motzkin word on $X$ if it has the properties that

(a) the number of $\Perp$ in the word equals the number of $\Perp$ in the word;

(b) counting from the left, the number of occurrence of $\Perp$ is always greater or equal to the number of occurrence of $\Perp$.

The set of all Motzkin words is denoted by $\mathcal{W}(X)$.

Example 3.5. Let $x, y, z$ be elements in $X$.

(a) The word $\llbracket \llbracket x \Downarrow \llbracket y \Perp \Perp z$ is a Motzkin word.

(b) the word $\llbracket x \rrbracket \llbracket y z$ is not a Motzkin word since it does not satisfy the first property.

(c) The word $x \rrbracket \llbracket y \llbracket z \Downarrow$ is not a Motzkin word since it does not satisfy the second property.

Define

$$
P: \mathcal{W}(X) \rightarrow \mathcal{W}(X), \quad P(m)=\lfloor m \rrbracket, m \in \mathcal{W}(X) .
$$

Then $\mathcal{W}(X)$ is an operated monoid. 
Theorem 3.6. [11, Theorem 3.4] Let $Y$ be a set. Let

$$
\phi:=\phi_{Y}: \mathfrak{M}(Y) \rightarrow \mathcal{W}(Y), \quad \phi(u)=u, u \in Y,
$$

be the homomorphism of operated monoid defined by the universal property of the free operated monoid $\mathfrak{M}(Y)$. Then $\phi$ is an isomorphism.

3.3. $\vec{\star}$-bracketed words and $\vec{\star}$-Motzkin words. Let $X$ be a set. For $k \geqslant 1$, let $\star_{1}, \cdots, \star_{k}$ be distinct symbols not in $X$ and let $\vec{\star}=\left(\star_{1}, \cdots, \star_{k}\right)$. Denote $X^{\vec{\star}}=X \cup\left\{\star_{1}, \cdots, \star_{k}\right\}$.

Definition 3.7. A $\vec{\star}$-bracketed word on $X$ is defined to be a bracketed word in the operated monoid $\mathfrak{M}\left(X^{\vec{\star}}\right)$ with exactly one occurrence of $\star_{i}$ for each $i=1, \cdots, k$. The set of all $\vec{\star}$-bracketed words on $X$ is denoted by $\mathfrak{M}^{\vec{\star}}(X)$. When $k=1$ and 2 , we denote $\mathfrak{M}^{\vec{\star}}(X)$ by $\mathfrak{M}^{\star}(X)$ and $\mathfrak{M}^{\star_{1}, \star_{2}}(X)$ respectively.

Definition 3.8. For $q \in \mathfrak{M}^{\vec{\star}}(X)$ and $s_{1}, \cdots, s_{k} \in \mathfrak{M}(X)$, we define

$$
\left.q\right|_{\vec{s}}:=\left.q\right|_{s_{1}, \cdots, s_{k}}
$$

to be the bracketed word in $\mathfrak{M}(X)$ obtained by replacing the letter $\star_{i}$ in $q$ by $s_{i}$ for $1 \leqslant i \leqslant k$. A $\left(s_{1}, \cdots, s_{k}\right)$-bracketed word on $X$ is a bracketed word of the form Eq. (G) for some $q \in \mathfrak{M}^{\vec{\star}}(X)$.

We next introduce the concept of a $\vec{\star}$-Motzkin word on $X$.

Definition 3.9. A word in $M\left(X^{\vec{\star}} \cup\{\amalg, \Perp\}\right)$ is called a $\vec{\star}$-Motzkin word on $X$ if it is in the intersection $M^{\vec{\star}}(X \cup\{\Perp, \Downarrow\}) \cap \mathcal{W}\left(X^{\vec{\star}}\right)$ or, more precisely, if it has the properties that

(a) the number of $\Perp$ in the word equals the number of $\Perp$ in the word;

(b) counting from the left, the number of occurrence of $\amalg$ is always greater or equal to the number of occurrence of $\Perp$;

(c) for each $1 \leqslant i \leqslant k$, the letter $\star_{i}$ appears exactly once in the word.

The set of all $\vec{\star}$-Motzkin words is denoted by $\mathcal{W}^{\vec{\star}}(X)$.

Taking $Y=X^{\star}$ in Theorem 3.6, we obtain

Corollary 3.10. There is a unique isomorphism

$$
\phi_{X^{\star}}: \mathfrak{M}\left(X^{\vec{\star}}\right) \rightarrow \mathcal{W}\left(X^{\vec{\star}}\right)
$$

of operated monoids such that $\phi_{X^{\star}}(a)=$ a for all $a \in X^{\vec{\star}}$. In particular, $\phi_{X^{\star}}\left(\star_{i}\right)=\star_{i}$ for $1 \leqslant i \leqslant k$.

Proposition 3.11. The isomorphism $\phi_{X^{\star}}$ restricts to a bijection

$$
\phi_{\vec{\star}}: \mathfrak{M}^{\vec{\star}}(X) \rightarrow \mathcal{W}^{\vec{\star}}(X) .
$$

Proof. Since the bijection $\phi_{X^{\star}}$ sends $\star_{i}$ to $\star_{i}, 1 \leqslant i \leqslant k$, an element $w \in \mathfrak{M}\left(X^{\vec{\star}}\right)$ is in $\mathfrak{M}^{\vec{\star}}(X)$ if and only if $\phi_{X^{\star}}(w) \in \mathcal{W}\left(X^{\vec{\star}}\right)$ is in $\mathcal{W}^{\vec{\star}}(X)$, hence the proposition.

\section{Relative LOCATIONS IN BRACKETED WORDS AND MOTZKIN WORDS}

In this section, we first define the relative locations of bracketed words and Motzkin words by using $\vec{\star}$-bracketed words and $\vec{\star}$-Motzkin words in Section 4.1. The relationship between the two kinds of relative locations is established in Section 4.2 and is used to prove the main theorem in Sections 4.3 . 


\subsection{Placements in bracketed words and Motzkin words.}

Definition 4.1. Let $X$ be a set. Let $f, s$ be in $\mathfrak{M}(X)$. A placement of $s$ in $f$ (by $q$ ) is a pair $\mathfrak{p}:=(s, q)$ with $q \in \mathfrak{M}^{\star}(X)$ such that $f=\left.q\right|_{s}$. We then call $s$ a bracketed subword of $f$.

The relative locations between two placements in a bracketed word can now be defined in the same way as those in an ordinary word.

Definition 4.2. Two placements $\left(s_{1}, q_{1}\right)$ and $\left(s_{2}, q_{2}\right)$ in $f \in \mathfrak{M}(X)$ are called

(a) separated if there exists an element $q$ in $\mathfrak{M}^{\star_{1}, \star_{2}}(X)$ such that $\left.q_{1}\right|_{\star_{1}}=\left.q\right|_{\star_{1}, s_{2}},\left.q_{2}\right|_{\star_{2}}=\left.q\right|_{s_{1}, \star_{2}}$ and $f=\left.q\right|_{s_{1}, s_{2}}$;

(b) nested if there exists an element $q$ in $\mathfrak{M}^{\star}(X)$ such that $q_{1}=\left.q_{2}\right|_{q}$ or $q_{2}=\left.q_{1}\right|_{q}$;

(c) intersecting if there exist an element $q$ in $\mathfrak{M}^{\star}(X)$ and elements $a, b, c$ in $\mathfrak{M}(X) \backslash\{1\}$ such that

(i) either $f=\left.q\right|_{a b c}, q_{1}=\left.q\right|_{\star c}, q_{2}=\left.q\right|_{a \star}$;

(ii) or $f=\left.q\right|_{a b c}, q_{1}=\left.q\right|_{a \star}, q_{2}=\left.q\right|_{\star c}$.

A bracketed word $s$ might appear in a bracketed word $f$ with multiple placements with different relative locations with respect to another bracketed subword.

Example 4.3. Let $f=\lfloor\lfloor a b c\rfloor a b\rfloor \in \mathfrak{M}(X)$. Let $s_{1}=\lfloor a b c\rfloor$ and let $s_{2}=a b$. Then we obtain the placements $\left(s_{1}, q_{1}\right),\left(s_{2}, q_{21}\right)$ and $\left(s_{2}, q_{22}\right)$, where $q_{1}=\lfloor\star a b\rfloor, q_{21}=\lfloor\lfloor\star c\rfloor a b\rfloor$ and $q_{22}=\lfloor\lfloor a b c\rfloor \star\rfloor$. Then the placements $\left(s_{1}, q_{1}\right)$ and $\left(s_{2}, q_{21}\right)$ are nested since $q_{21}=\left.q_{1}\right|_{q}$, where $q=\lfloor\star c\rfloor$. However the placements $\left(s_{1}, q_{1}\right)$ and $\left(s_{2}, q_{22}\right)$ are separated.

We next describe the relative locations of two placements of bracketed words in terms of Motzkin words. By Definition 3.4, we obtain

Lemma 4.4. Let $u$ be in $\mathcal{W}(X)$ and $p$ be in $\mathcal{W}^{\star}(X)$. Then $\left.p\right|_{u}$ is in $\mathcal{W}(X)$.

Taking $Z=X \cup\{\lfloor, \Perp\}$ in Definition 2.3, we have

Definition 4.5. Let $w$ and $u$ be Motzkin words in $\mathcal{W}(X)$. A placement of $u$ in $w$ (by $p$ ) is a pair $(u, p)$ with $p \in \mathcal{W}^{\star}(X)$ such that $\left.p\right|_{u}=w$. We then call $u$ a Motzkin subword of $w$.

By Definition 3.4, the set $\mathcal{W}^{\star_{1}, \star_{2}}(X)$ of $\left(\star_{1}, \star_{2}\right)$-Motzkin words is a subset of $M^{\star_{1}, \star_{2}}(X \cup\{\amalg, \|\})$. Thus as a special case of Definition 2.5, we obtain the definition of two placements $\left(u_{1}, p_{1}\right)$ and $\left(u_{2}, p_{2}\right)$ in $w$ being separated or nested or intersecting.

4.2. Relationship between relative locations. We now establish the relationship between placements in bracketed words and placements in Motzkin words.

Proposition 4.6. Let $X$ be a set and let $\left\{\star_{1}, \cdots, \star_{k}\right\}$ be symbols not in $X$. Let $\phi: \mathfrak{M}(X) \rightarrow \mathcal{W}(X)$ be the isomorphism of operated monoids in Theorem 3.6, and let $\phi_{\vec{\star}}: \mathfrak{M}^{\vec{\star}}(X) \rightarrow \mathcal{W}^{\vec{\star}}(X)$ be the bijection in Proposition 3.11 .

(a) Let $q \in \mathfrak{M}^{\vec{\star}}(X)$ and $s_{1}, \cdots, s_{k} \in \mathfrak{M}(X)$. Then $\phi\left(\left.q\right|_{s_{1}, \cdots, s_{k}}\right)=\left.\phi_{\vec{\star}}(q)\right|_{\phi\left(s_{1}\right), \cdots, \phi\left(s_{k}\right)}$.

(b) Let $p \in \mathcal{W}^{\vec{\star}}(X)$ and $u_{1}, \cdots, u_{k} \in \mathcal{W}(X)$. Then $\phi^{-1}\left(\left.p\right|_{u_{1}, \cdots, u_{k}}\right)=\left.\phi_{\vec{\star}}^{-1}(p)\right|_{\phi^{-1}\left(u_{1}\right), \cdots, \phi^{-1}\left(u_{k}\right)}$.

Proof. (a) We prove a more general result. Define

$\overline{\mathfrak{M}}^{\vec{\star}}(X):=\left\{q \in \mathfrak{M}\left(X^{\vec{\star}}\right) \mid\right.$ for each $1 \leqslant i \leqslant k, \star_{i}$ appears at most once in $\left.q\right\} \subseteq \mathfrak{M}\left(X^{\vec{\star}}\right)$. 
For $q \in \overline{\mathfrak{M}}^{\vec{\star}}(X)$ and $s_{1}, \cdots, s_{k} \in \mathfrak{M}(X)$, define $\left.q\right|_{s_{1}, \cdots, s_{k}}$ to be the bracketed word by replacing the $\star_{i}$ in $q$, if there is any, by $s_{i}, 1 \leqslant i \leqslant k$. Thus in particular, $\left.q\right|_{s_{1}, \cdots, s_{k}}=\left.q\right|_{s_{1}}$ if $q \in \mathfrak{M}^{\star_{1}}(X)$ and $\left.q\right|_{s_{1}, \cdots, s_{k}}=q$ if $q \in \mathfrak{M}(X)$. In general, $\left.q\right|_{s_{1}, \cdots, s_{k}}=\left.q\right|_{s_{i_{1}}, \cdots, s_{i m}}$ if only $\star_{i_{1}}, \cdots, \star_{i_{m}}$ from $\left\{\star_{1}, \cdots, \star_{k}\right\}$ appear in $q$.

Similarly define

$$
\overrightarrow{\mathcal{W}}^{\vec{\star}}(X):=\left\{p \in \mathcal{W}\left(X^{\vec{\star}}\right) \mid \text { for each } 1 \leqslant i \leqslant k, \star_{i} \text { appears at most once in } p\right\} \subseteq \mathcal{W}\left(X^{\vec{\star}}\right),
$$

and define $\left.p\right|_{u_{1}, \cdots, u_{k}}$ for $p \in \overline{\mathcal{W}}^{\vec{\star}}(X)$ and $u_{1}, \cdots, u_{k} \in \mathcal{W}(X)$.

With these notations, if $q$ is in $\overline{\mathfrak{M}}^{\vec{*}}(X)$ and $q=q_{1} q_{2}$ with $q_{1}, q_{2} \in \overline{\mathfrak{M}}^{\vec{*}}(X)$, then we have $\left.q\right|_{\vec{s}}=\left.\left.q_{1}\right|_{\vec{s}} q_{2}\right|_{\vec{s}}$. Further the bijection $\phi_{X^{\vec{x}}}$ in Corollary 3.10 restricts to a bijection

$$
\bar{\phi}_{\vec{\star}}: \overline{\mathfrak{M}}^{\vec{\star}}(X) \rightarrow \overline{\mathcal{W}}^{\vec{\star}}(X)
$$

by the same argument as Proposition 3.11 .

Claim 4.7. Let $q \in \overline{\mathfrak{M}}^{\vec{*}}(X)$ and $s_{1}, \cdots, s_{k} \in \mathfrak{M}(X)$. Then $\phi\left(\left.q\right|_{s_{1}, \cdots, s_{k}}\right)=\left.\bar{\phi}_{\vec{\star}}(q)\right|_{\phi\left(s_{1}\right), \cdots, \phi\left(s_{k}\right)}$.

Proof. We prove the claim by induction on the depth of $q \in \overline{\mathfrak{M}}^{\vec{*}}(X)$ defined in Eq. (5).

If the depth of $q$ is 0 , then $q \in \bar{M}^{\vec{\star}}(X)$ and hence $q=z_{1} \star_{i_{1}} z_{2} \star_{i_{2}} \cdots z_{m} \star_{i_{m}} z_{m+1}$ with $z_{1}, \cdots, z_{m+1} \in$ $M(X)$ and $\star_{i_{1}}, \cdots, \star_{i_{m}} \in\left\{\star_{1}, \cdots, \star_{k}\right\}$. Then we have

$$
\phi\left(\left.q\right|_{\vec{s}}\right)=\phi\left(z_{1} s_{i_{1}} \cdots z_{m} s_{i_{m}} z_{m+1}\right)=\phi\left(z_{1}\right) \phi\left(s_{i_{1}}\right) \cdots \phi\left(z_{m}\right) \phi\left(s_{i_{m}}\right) \phi\left(z_{m+1}\right)=\left.\bar{\phi}_{\vec{\star}}(q)\right|_{\phi(\vec{s})},
$$

as needed. Here we have used the abbreviations $\vec{s}=\left(s_{1}, \cdots, s_{k}\right)$ and $\phi(\vec{s})=\left(\phi\left(s_{1}\right), \cdots, \phi\left(s_{k}\right)\right)$.

Assume that the claim has been proved for $q \in \overline{\mathfrak{M}}^{\vec{*}}(X)$ with depth less or equal to $n \geqslant 0$ and consider a $q$ with depth $n+1$.

Note that $\phi$ and $\phi_{X^{\vec{x}}}$ (and hence $\bar{\phi}_{\vec{\star}}$ ) are multiplicative. Thus for $q=q_{1} q_{2}$ with $q, q_{1}, q_{2} \in$ $\overline{\mathfrak{M}}^{\vec{\star}}(X)$, if we can prove that $\phi\left(\left.q_{i}\right|_{\vec{s}}\right)=\left.\bar{\phi}_{\vec{\star}}\left(q_{i}\right)\right|_{\phi(\vec{s})}, i=1,2$, then we also have

$$
\phi\left(\left.q\right|_{\vec{s}}\right)=\phi\left(q_{1} \mid \vec{s}\right) \phi\left(\left.q_{2}\right|_{\vec{s}}\right)=\left.\left.\bar{\phi}_{\vec{\star}}\left(q_{1}\right)\right|_{\phi(\vec{s})} \bar{\phi}_{\vec{\star}}\left(q_{2}\right)\right|_{\phi(\vec{s})}=\left.\left(\bar{\phi}_{\vec{\star}}\left(q_{1}\right) \bar{\phi}_{\vec{\star}}\left(q_{2}\right)\right)\right|_{\phi(\vec{s})}=\left.\bar{\phi}_{\vec{\star}}(q)\right|_{\phi(\vec{s})} .
$$

Therefore, we only need to complete the induction when $q$ is of depth $n+1$ and is indecomposable in $\overline{\mathfrak{M}}^{\vec{\star}}(X)$, that is, $q$ is not the product of two elements in $\overline{\mathfrak{M}}^{\vec{\star}}(X) \subseteq \mathfrak{M}\left(X^{\vec{\star}}\right)=M\left(X^{\vec{\star}} \cup\left\lfloor\mathfrak{M}\left(X^{\vec{\star}}\right)\right\rfloor\right)$ (see Eq. (丑). Then $q$ is either in $X \cup\left\{\star_{1}, \cdots, \star_{k}\right\}$ or is of the form $\lfloor\widetilde{q}\rfloor$ with $\widetilde{q} \in \overline{\mathfrak{M}}^{\star}(X)$. In the first case, $q$ is of depth 0 and has been proved above. In the second case, $\widetilde{q}$ has depth $n$. Note that $\phi$ and $\phi_{\vec{\star}}$ (and hence $\bar{\phi}_{\vec{\star}}$ ) are compatible with the brackets. So together with the induction hypothesis, we have

$$
\left.\phi\left(\left.q\right|_{\vec{s}}\right)=\phi\left(\left.\lfloor\widetilde{q}\rfloor\right|_{\vec{s}}\right)=\phi\left(\left\lfloor\left.\widetilde{q}\right|_{\vec{s}}\right\rfloor\right)=\left\lfloor\phi\left(\left.\widetilde{q}\right|_{\vec{s}}\right)\right\rfloor=\left\lfloor\left.\bar{\phi}_{\vec{\star}}(\widetilde{q})\right|_{\phi(\vec{s})}\right\rfloor=\left(\left\lfloor\bar{\phi}_{\vec{\star}} \widetilde{q}\right)\right\rfloor\right)\left.\right|_{\phi(\vec{s})}=\left.\left(\bar{\phi}_{\vec{\star}}(\lfloor\widetilde{q}\rfloor)\right)\right|_{\phi(\vec{s})}=\left.\bar{\phi}_{\vec{\star}}(q)\right|_{\phi(\vec{s})} .
$$

This completes the inductive proof of the claim.

Then Item (d) is a special case of the claim since the restriction of $\bar{\phi}_{\vec{\star}}$ to $\mathfrak{M}^{\vec{\star}}(X)$ is $\phi_{\vec{\star}}$. (b) Denote $\vec{u}=\left(u_{1}, \cdots, u_{k}\right)$ and $\phi^{-1}(\vec{u})=\left(\phi^{-1}\left(u_{1}\right), \cdots, \phi^{-1}\left(u_{k}\right)\right)$. By Item (a) and the bijectivity of $\phi$ and $\phi_{\vec{\star}}$, we have

$$
\phi^{-1}\left(\left.p\right|_{\vec{u}}\right)=\phi^{-1}\left(\left.\phi_{\vec{\star}}\left(\phi_{\vec{\star}}^{-1}(p)\right)\right|_{\phi\left(\phi^{-1}(\vec{u})\right)}\right)=\phi^{-1}\left(\phi\left(\left.\phi_{\vec{\star}}^{-1}(p)\right|_{\phi^{-1}(\vec{u})}\right)\right)=\left.\phi_{\vec{\star}}^{-1}(p)\right|_{\phi^{-1}(\vec{u})} .
$$

This is what we need. 
By Proposition 4.6, we immediately have

Corollary 4.8. Let $f$ be in $\mathfrak{M}(X)$. Then $(s, q)$ is a placement of $s$ in $f$ if and only if $\left(\phi(s), \phi_{\star}(q)\right)$ is a placement of $\phi(s)$ in $\phi(f) \in \mathcal{W}(X)$.

Proof. The pair $(s, q)$ is a placement of $s$ in $f$ if and only if $f=\left.q\right|_{s}$, which holds if and only if $\phi(f)=\left.\phi_{\star}(q)\right|_{\phi(s)}$, which holds if and only if $\left(\phi(s), \phi_{\star}(q)\right)$ is a placement of $\phi(s)$ in $\phi(f)$.

We also have

Corollary 4.9. Let $q_{1}, q_{2}$ and $q$ be $\vec{\star}$-bracketed words in $\mathfrak{M}^{\star}(X)$. Then $q_{1}=\left.q_{2}\right|_{q}$ if and only if $\phi_{\star}\left(q_{1}\right)=\left.\phi_{\star}\left(q_{2}\right)\right|_{\phi_{\star}(q)}$ in $\mathcal{W}^{\star}(X)$.

Proof. To be precise, denote the isomorphism and the bijection in Proposition 4.6, in the case of $k=1$, by

$$
\phi:=\phi_{X}: \mathfrak{M}(X) \rightarrow \mathcal{W}(X), \quad \phi_{\star_{1}}:=\phi_{X, \star_{1}}: \mathfrak{M}^{\star_{1}}(X) \rightarrow \mathcal{W}^{\star_{1}}(X) .
$$

Also denote $X^{\prime}:=X \cup\{\star\}$ where $\star$ is a symbol not in $X \cup\left\{\star_{1}\right\}$. Then $\left.\phi_{X^{\prime}}\right|_{\mathfrak{M}^{\star}(X)}=\phi_{X, \star}$.

Let $q_{1}, q_{2}, q \in \mathfrak{M}^{\star}(X)$ be given. Define $q_{2}^{\prime}:=\left.q_{2}\right|_{\star \rightarrow \star_{1}} \in \mathfrak{M}^{\star_{1}}(X) \subseteq \mathfrak{M}^{\star_{1}}\left(X^{\prime}\right)$. We also have $q_{1}, q \in \mathfrak{M}^{\star}(X) \subseteq \mathfrak{M}\left(X^{\prime}\right)$. Then $q_{1}=\left.q_{2}\right|_{q}$ means $q_{1}=\left.q_{2}^{\prime}\right|_{q}$ in $\mathfrak{M}\left(X^{\prime}\right)$. Then applying Proposition 4.6.(a) to the set $X^{\prime}$ with $k=1$, we have

$$
\phi_{\star}\left(q_{1}\right)=\phi_{X, \star}\left(q_{1}\right)=\phi_{X^{\prime}}\left(q_{1}\right)=\phi_{X^{\prime}}\left(\left.q_{2}^{\prime}\right|_{q}\right)=\left.\phi_{X^{\prime}, \star_{1}}\left(q_{2}^{\prime}\right)\right|_{\phi_{X^{\prime}}(q)}=\left.\phi_{\star_{1}}\left(q_{2}^{\prime}\right)\right|_{\phi_{\star}(q)} .
$$

But $\left.q_{2}^{\prime}\right|_{\star}=q_{2}$, so $\left.\phi_{\star}\left(q_{2}^{\prime}\right)\right|_{\phi_{\star}(q)}=\left.\phi_{\star}\left(q_{2}\right)\right|_{\phi_{\star}(q)}$. Hence

$$
\phi_{\star}\left(q_{1}\right)=\left.\phi_{\star}\left(q_{2}\right)\right|_{\phi_{\star}(q)} .
$$

Conversely, if $\phi_{\star}\left(q_{1}\right)=\left.\phi_{\star}\left(q_{2}\right)\right|_{\phi_{\star}(q)}$, then we have $\phi_{\star}\left(q_{1}\right)=\left.\phi_{\star_{1}}\left(q_{2}^{\prime}\right)\right|_{\phi_{\star}(q)}$, where $q_{2}^{\prime}=\left.q_{2}\right|_{\star_{1}}$. Then with the notations as above, applying Proposition 4.6.(b), we have

$$
q_{1}=\phi_{\star}^{-1}\left(\phi_{\star}\left(q_{1}\right)\right)=\phi_{\star}^{-1}\left(\left.\phi_{\star_{1}}\left(q_{2}^{\prime}\right)\right|_{\phi(q)}\right)=\left.\phi_{\star_{1}}^{-1}\left(\phi_{\star_{1}}\left(q_{2}^{\prime}\right)\right)\right|_{\phi_{\star}^{-1}\left(\phi_{\star}(q)\right)}=\left.q_{2}^{\prime}\right|_{q}=\left.q_{2}\right|_{q}
$$

Theorem 4.10. Let $f$ be a bracketed word in $\mathfrak{M}(X)$. Let $\left(s_{1}, q_{1}\right)$ and $\left(s_{2}, q_{2}\right)$ be placements in $f$. Then

(a) the pairs $\left(s_{1}, q_{1}\right)$ and $\left(s_{2}, q_{2}\right)$ are separated in $f$ if and only if the pairs $\left(\phi\left(s_{1}\right), \phi_{\star}\left(q_{1}\right)\right)$ and $\left(\phi\left(s_{2}\right), \phi_{\star}\left(q_{2}\right)\right)$ are separated in $\phi(f)$;

(b) the pairs $\left(s_{1}, q_{1}\right)$ and $\left(s_{2}, q_{2}\right)$ are nested in $f$ if and only if the pairs $\left(\phi\left(s_{1}\right), \phi_{\star}\left(q_{1}\right)\right)$ and $\left(\phi\left(s_{2}\right), \phi_{\star}\left(q_{2}\right)\right)$ are nested in $\phi(f)$;

(c) the pairs $\left(s_{1}, q_{1}\right)$ and $\left(s_{2}, q_{2}\right)$ are intersecting in $f$ if and only if the pairs $\left(\phi\left(s_{1}\right), \phi_{\star}\left(q_{1}\right)\right)$ and $\left(\phi\left(s_{2}\right), \phi_{\star}\left(q_{2}\right)\right)$ are intersecting in $\phi(f)$.

Proof. Let $\left(s_{1}, q_{1}\right)$ and $\left(s_{2}, q_{2}\right)$ be placements in $f$. Then by Corollary $4.8,\left(\phi\left(s_{1}\right), \phi_{\star}\left(q_{1}\right)\right)$ and $\left(\phi\left(s_{2}\right), \phi_{\star}\left(q_{2}\right)\right)$ are placements in $\phi(f)$.

(a) The placements $\left(s_{1}, q_{1}\right)$ and $\left(s_{2}, q_{2}\right)$ in $f$ are separated if and only if there exists an element $q$ in $\mathfrak{M i}^{\star_{1}, \star_{2}}(X)$ such that

$$
\left.q_{1}\right|_{\star_{1}}=\left.q\right|_{\star_{1}, s_{2}},\left.\quad q_{2}\right|_{\star_{2}}=\left.q\right|_{s_{1}, \star_{2}}, \quad f=\left.q\right|_{s_{1}, s_{2}} .
$$

By Proposition 4.6, this is so if and only if

$$
\left.\phi_{\star}\left(q_{1}\right)\right|_{\star_{1}}=\left.\phi_{\star_{1}, \star_{2}}(q)\right|_{\star_{1}, \phi\left(s_{2}\right)},\left.\quad \phi_{\star}\left(q_{2}\right)\right|_{\star_{2}}=\left.\phi_{\star_{1}, \star_{2}}(q)\right|_{\phi\left(s_{1}\right), \star_{2}}, \quad \phi(f)=\left.\phi_{\star_{1}, \star_{2}}(q)\right|_{\phi\left(s_{1}\right), \phi\left(s_{2}\right)} .
$$


If this is true, then $\left(\phi\left(s_{1}\right), \phi_{\star}\left(q_{1}\right)\right)$ and $\left(\phi\left(s_{2}\right), \phi_{\star}\left(q_{2}\right)\right)$ are separated in $\phi(f)$. Conversely, if the placements $\left(\phi\left(s_{1}\right), \phi_{\star}\left(q_{1}\right)\right)$ and $\left(\phi\left(s_{2}\right), \phi_{\star}\left(q_{2}\right)\right)$ are separated in $\phi(f)$, then there exists an element $p$ in $\mathcal{W}^{\star_{1}, \star_{2}}(X)$ such that

$$
\left.\phi_{\star}\left(q_{1}\right)\right|_{\star_{1}}=\left.p\right|_{\star_{1}, \phi\left(s_{2}\right)},\left.\quad \phi_{\star}\left(q_{2}\right)\right|_{\star_{2}}=\left.p\right|_{\phi\left(s_{1}\right), \star_{2}}, \quad \phi(f)=\left.p\right|_{\phi\left(s_{1}\right), \phi\left(s_{2}\right)} .
$$

Since $\phi_{\star_{1}, \star_{2}}: \mathfrak{M}^{\star_{1}, \star_{2}}(X) \rightarrow \mathcal{W}^{\star_{1}, \star_{2}}(X)$ is bijective, there is $q \in \mathfrak{M}^{\star_{1}, \star_{2}}(X)$ such that $\phi_{\star_{1}, \star_{2}}(q)=p$. Thus Eq. (7) holds and hence $\left(s_{1}, q_{1}\right)$ and $\left(s_{2}, q_{2}\right)$ are separated in $f$.

(b) The placements $\left(s_{1}, q_{1}\right)$ and $\left(s_{2}, q_{2}\right)$ are nested in $f$ if and only if there exists an element $q$ in $\mathfrak{M}^{\star}(X)$ such that $q_{1}=\left.q_{2}\right|_{q}$ or $q_{2}=\left.q_{1}\right|_{q}$. By Corollary 4.9, this holds if and only if

$$
\phi_{\star}\left(q_{1}\right)=\left.\phi_{\star}\left(q_{2}\right)\right|_{\phi_{\star}(q)} \quad \text { or } \quad \phi_{\star}\left(q_{2}\right)=\left.\phi_{\star}\left(q_{1}\right)\right|_{\phi_{\star}(q)} .
$$

If this is true, then $\left(\phi\left(s_{1}\right), \phi_{\star}\left(q_{1}\right)\right)$ and $\left(\phi\left(s_{2}\right), \phi_{\star}\left(q_{2}\right)\right)$ are nested. Conversely, if $\left(\phi\left(s_{1}\right), \phi_{\star}\left(q_{1}\right)\right)$ and $\left(\phi\left(s_{2}\right), \phi_{\star}\left(q_{2}\right)\right)$ are nested, then there is $p \in \mathcal{W}^{\star}(X)$ such that

$$
\phi_{\star}\left(q_{1}\right)=\left.\phi_{\star}\left(q_{2}\right)\right|_{p} \quad \text { or } \quad \phi_{\star}\left(q_{2}\right)=\left.\phi_{\star}\left(q_{1}\right)\right|_{p} .
$$

Since $\phi_{\star}: \mathfrak{M}^{\star}(X) \rightarrow \mathcal{W}^{\star}(X)$ is bijective, there is $q \in \mathfrak{M}^{\star}(X)$ such that $\phi_{\star}(q)=p$. Thus Eq. (8) holds and hence $\left(s_{1}, q_{1}\right)$ and $\left(s_{2}, q_{2}\right)$ are nested in $f$.

(C) The placements $\left(s_{1}, q_{1}\right)$ and $\left(s_{2}, q_{2}\right)$ are intersecting in $f$ if and only if there exist $q$ in $\mathfrak{M}^{\star}(X)$ and $a, b, c$ in $\mathfrak{M}(X) \backslash\{1\}$ such that

$$
f=\left.q\right|_{a b c}, \quad q_{1}=\left.q\right|_{\star c}, \quad q_{2}=\left.q\right|_{a \star},
$$

or

$$
f=\left.q\right|_{a b c}, \quad q_{1}=\left.q\right|_{a \star}, \quad q_{2}=\left.q\right|_{\star c} .
$$

By Theorem 3.6, Proposition 4.6 and Corollary 4.9, this is so if and only if

$$
\phi(f)=\left.\phi_{\star}(q)\right|_{\phi(a) \phi(b) \phi(c)}, \quad \phi_{\star}\left(q_{1}\right)=\left.\phi_{\star}(q)\right|_{\phi(a) \star}, \quad \phi_{\star}\left(q_{2}\right)=\left.\phi_{\star}(q)\right|_{\star \phi(c)},
$$

or

$$
\phi(f)=\left.\phi_{\star}(q)\right|_{\phi(a) \phi(b) \phi(c)}, \quad \phi_{\star}\left(q_{1}\right)=\left.\phi_{\star}(q)\right|_{\star \phi(c)}, \quad \phi_{\star}\left(q_{2}\right)=\left.\phi_{\star}(q)\right|_{\phi(a) \star} .
$$

If this is true, then $\left(\phi\left(s_{1}\right), \phi_{\star}\left(q_{1}\right)\right)$ and $\left(\phi\left(s_{2}\right), \phi_{\star}\left(q_{2}\right)\right)$ are intersecting since $\phi(a), \phi(b), \phi(c) \neq 1$.

Conversely, if $\left(\phi\left(s_{1}\right), \phi_{\star}\left(q_{1}\right)\right)$ and $\left(\phi\left(s_{2}\right), \phi_{\star}\left(q_{2}\right)\right)$ are intersecting in $\phi(f)$, then there exist $p$ in $\mathcal{W}^{\star}(X)$ and $\alpha, \beta, \gamma$ in $\mathcal{W}(X) \backslash\{1\}$ such that

$$
\phi(f)=\left.p\right|_{\alpha \beta \gamma}, \quad \phi_{\star}\left(q_{1}\right)=\left.\phi_{\star}(q)\right|_{\alpha \star}, \quad \phi_{\star}\left(q_{2}\right)=\left.\phi_{\star}(q)\right|_{\star \gamma},
$$

or

$$
\phi(f)=\left.p\right|_{\alpha \beta \gamma}, \quad \phi_{\star}\left(q_{1}\right)=\left.\phi_{\star}(q)\right|_{\star \gamma}, \quad \phi_{\star}\left(q_{2}\right)=\left.\phi_{\star}(q)\right|_{\alpha \star} .
$$

By the bijectivity of $\phi$ and $\phi_{\star}$, there are $q \in \mathfrak{M}^{\star}(X)$ and $a, b, c \in \mathfrak{M}(X) \backslash\{1\}$ such that $p=\phi_{\star}(q)$ and $\alpha=\phi(a), \beta=\phi(b), \gamma=\phi(c)$. Then Eqs. (9) or (10) hold. This shows that $\left(s_{1}, q_{1}\right)$ and $\left(s_{2}, q_{2}\right)$ are intersecting in $f$. 
4.3. Relative locations of bracketed subwords. Now we are ready to prove our main theorem on the classification of relative locations of two bracketed subwords (placements) in a bracketed word.

Theorem 4.11. (Main Theorem) Let $f$ be a bracketed word in $\mathfrak{M}(X)$. For any two placements $\left(s_{1}, q_{1}\right)$ and $\left(s_{2}, q_{2}\right)$ in $f$, exactly one of the following is true:

(a) $\left(s_{1}, q_{1}\right)$ and $\left(s_{2}, q_{2}\right)$ are separated;

(b) $\left(s_{1}, q_{1}\right)$ and $\left(s_{2}, q_{2}\right)$ are nested;

(c) $\left(s_{1}, q_{1}\right)$ and $\left(s_{2}, q_{2}\right)$ are intersecting.

Proof. By Theorem 2.11, the statement of the theorem holds when $\left(s_{1}, q_{1}\right)$ and $\left(s_{2}, q_{2}\right)$ are replaced by the two placements $\left(\phi\left(s_{1}\right), \phi_{\star}\left(q_{1}\right)\right)$ and $\left(\phi\left(s_{2}\right), \phi_{\star}\left(q_{2}\right)\right)$ in the word $\phi(f) \in \mathcal{W}(X) \subseteq$ $M(X \cup\{\Perp, \Perp\})$. Then by Theorem 4.10, the statement holds for $\left(s_{1}, q_{1}\right)$ and $\left(s_{2}, q_{2}\right)$.

Acknowledgements: L. Guo acknowledges support from NSF grant DMS 1001855. S. Zheng thanks support from NSFC grant 11201201 and Fundamental Research Funds for the Central Universities lzujbky-2013-8.

\section{REFERENCES}

[1] L. Alonso, Uniform generation of a Motzkin word, Theor. Comput.Sci. 134 (1994) 529-536. 6

[2] F. Baader and T. Nipkow, Term Rewriting and All That. Cambridge U. P., Cambridge, 1998.11.2

[3] C. Bogner and S. Weinzierl, Blowing up Feynman integrals, Nucl. Phys. Proc. Suppl. 183 (2008) 256261. 2

[4] L. A. Bokut, Y. Chen and Y. Chen, Composition-Diamond lemma for tensor product of free algebras, $J$. Algebra, 323 (2010), 2520-2537. 目

[5] L. A. Bokut, Y. Chen and X. Deng, Gröbner-Shirshov bases for Rota-Baxter algebras, Siberian Math. J. 51 (2010), 978-988. 目

[6] L. A. Bokut, Y. Chen and Y. Li, Gröbner-Shirshov bases for categories, in "Operads and Universal Algebra", World Scientific Press, (2012) 1-23.

[7] L. A. Bokut, Y. Chen and J. Qiu, Greobner-Shirshov bases for associative algebras with multiple operators and free Rota-Baxter algebras, J. Pure Appl. Algebra, 214 (2010) 89-110.

[8] A. Connes and D. Kreimer, Hopf algebras, renormalization and concommutative geometry, Comm. Math. Phys. 199 (1998) 203-242. 2 , 7

[9] A. Connes and D. Kreimer, Renormalization in quantum field theory and the Riemann-Hilbert problem. I. The Hopf algebra structure of graphs and the main theorem, Comm. Math. Phys. 210 (2000) 249-273. 2

[10] R. Donaghey and L. W. Shapiro, Motzkin numbers, J. Combin. Theory Ser. A 23 (1977), 291-301.7

[11] L. Guo, Operated semigroups, Motzkin paths and rooted trees, J. Alger. Comb. 29 (2009), 35-62. 6 6 , 8

[12] L. Guo, An Introduction to Rota-Baxter Algebra, International Press and Higher Education Press, 2012. 2

[13] L. Guo, W. Sit and R. Zhang, Differemtail type operators and Gröbner-Shirshov bases, J. Symb. Comput. 52 (2013), 97-123. 2. 6

[14] D. Kreimer, On overlapping divergences, Comm. Math. Phys. 204 (1999) 669-689. 2 ,

[15] T. Krajewski and R. Wulkenhaar, On Kreimer's Hopf algebra structure of Feynman graphs, Eur. Phys. J. C, 7 (1999) 697-708. 目

[16] A. Sapounakis and P. Tsikouras, On $k$-colored Motzkin words, J. Inter Seq. 7 (2004), Article 04. 2.5. 6

[17] S. Zheng, X. Gao, L. Guo and W. Sit, Rota-Baxter type operators, rewriting systems and GröbnerShirshov bases, preprint. 目 
Department of Mathematics and Computer Science, Rutgers University, Newark, NJ 07102, USA

E-mail address: liguo@rutgers.edu

Department of Mathematics, Lanzhou University, Lanzhou, Gansu 730000, China

E-mail address: zheng2712801@163.com 